\title{
Bessel Function Self-Feedback Chaotic Neural Network Model and Applications
}

\author{
Yonggang Ye \\ School of Basic Science, Harbin University of Commerce \\ Harbin Heilongjiang \\ China 150028 \\ ye_yonggang@163
}

\begin{abstract}
In this paper, a new chaotic neural network model is proposed, we int oduce a Bessel function as self-feedback term in this model, Compared with other chabtic neural network model, owing to the Bessel function is a nonlinear function with good nature, and it has stronger function approximation ability, so that the novel chaotic network model has stronger traversal search ability. When it is applied to solve combinatorial optimization problems, the simulation results show that the network has better ability to avoid network convergence to local minima if the appropriate coefficient of expansion and the network has been taken, so the efficiency of network optimization capability is improved.
\end{abstract}

Keywords: chaotic neural network; Bessel functiôn, self-feedback; TSP problem

\section{Introduction}

In recent years, transiently chaotic neural networks (TCNN) with its complex nonlinear dynamical behaipr successfully and efficiently have solved the traveling salesman problem (TSP), and have been extensively research [1-6]. But in the reference [1], Chen-Aihara chaotic neuralnetwork is introduced through the simulated annealing mechanism, the network showed both transient and stable chaotic search behavior can converge at one point, so as to effectively overcome the shortcomings of the Hopfield network, greatly increase the accuracy and speed of the network to solve the traveling salesman problem andproves Chen-Aihara chaotic neural network can reach asymptotic stability, convergence of network provides a theoretical basis. Chaotic dynamics due to feedback is related to the different nature of the self-feedback function that can produce different chaotic traversal search, $\mathrm{Xu}$ Yaoqun, Sun Ming, Yang xueling put forward a nonlinear wavelet functions and trigonometric functions from transient chaotic neural feedback network model [7-9], such a model with a more sophisticated search capabilities in chaos chaotic search process. In this paper, based Bessel function well localized nature, by improving self- feedback term Chen-Aihara network, construct a Bessel function of the nonlinear self-feedback transiently chaotic neural network, using a unified framework for the construction of a theory Kwok network energy function and prove that the proposed network model under given conditions can be achieved asymptotically stable, continuous function optimization through simulation studies 10 cities TSP problem and show that, Bessel function self-feedback nonlinear transient chaotic neural network after selecting the appropriate scaling factor has a stronger ability to avoid network into a local minimum point. 


\section{Chaotic Neural Network of Bessel Function Self-feedback}

\subsection{Bessel Function}

In the standard second-order ordinary differential equations in the following, $y^{\prime \prime}(x)+p(x) y^{\prime}(x)+q(x) y(x)=0$, let $p(x)=\frac{1}{x}, q(x)=1-\frac{v^{2}}{x^{2}}$, it come to v-order Bessel equation $x^{2} y^{\prime \prime}(x)+x y^{\prime}(x)+\left(x^{2}-v^{2}\right) y(x)=0$, it can lead to v-order Bessel function in the process of solving method using power series:

$J_{v}(x)=\sum_{k=0}^{\infty}(-1)^{k} \frac{1}{k ! \Gamma(k+v+1)}\left(\frac{x}{2}\right)^{2 k+v}$

If let $v=-1,0,1$, then Bessel function of the image shown in Figure 1.

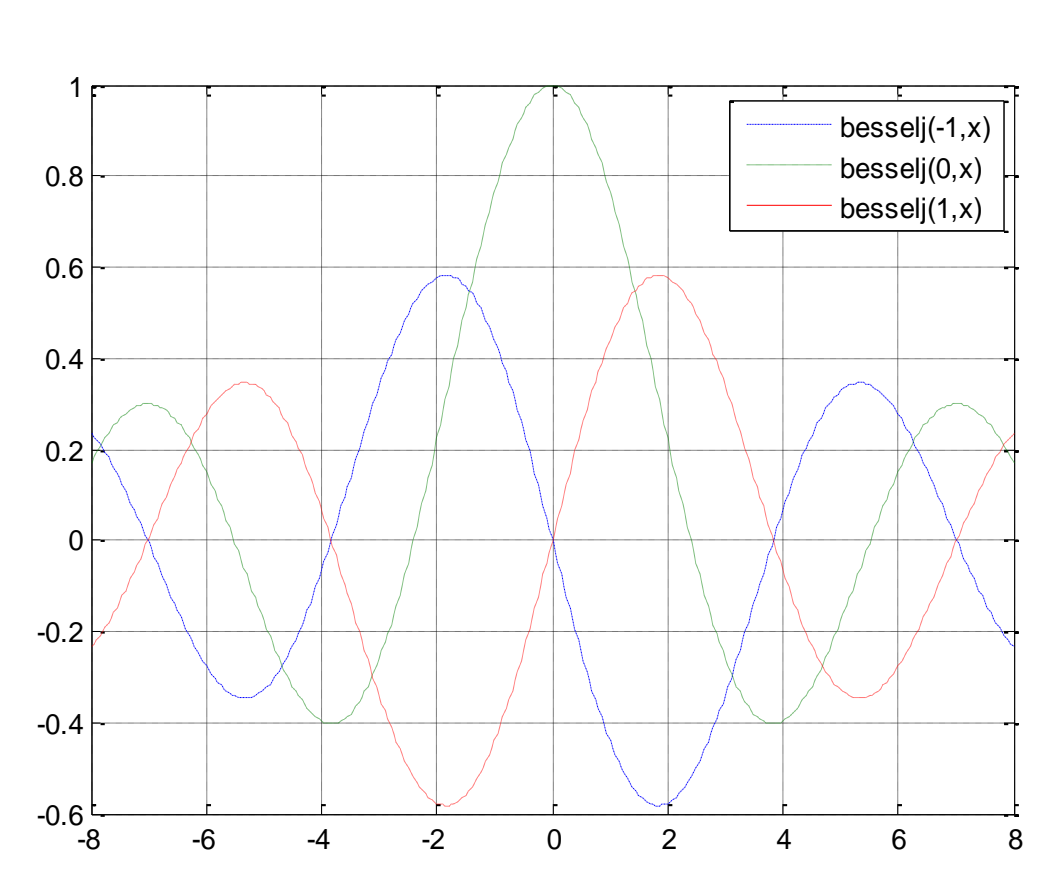

Figure 1. Figure of the Function When $v=-1,0,1$

It is obvioús when $v=-1$ or 1 , Bessel function is an odd function, and when $v=0$, Bessel function is an even function. These three Bessel function was shock attenuation and amplitude is less than or equal to 1 .

\subsection{Chaotic Neuron Model of Bessel Function Self-feedback}

Select $v=1$, we construct a self-feedback term chaotic neural network chaotic neuron model in the following:

$$
\begin{aligned}
& x(t)=\frac{1}{1+\exp (-y(t) / \varepsilon)} \\
& y(t+1)=k y(t)-z(t) g\left(x(t)-I_{0}\right)
\end{aligned}
$$




$$
\begin{aligned}
& g(x)=J_{1}(c x) \\
& z(t+1)=(1-\beta) z(t)
\end{aligned}
$$

where $x(\mathrm{t})$ is the output of the neuron at the time of $\mathrm{t} ; y(\mathrm{t})$ is the internal state of the neuron at the time of $\mathrm{t} ; I_{0}$ is a positive parameter; $k$ is a damping factor of nerve membrane( $0 \leq k \leq 1) ; \varepsilon$ is steepness parameter of the activation function; $\mathrm{z}(\mathrm{t})$ is the self-feedback connection weight; $\beta$ is the damping factor of $\mathrm{z}(\mathrm{t}) ; c$ is the scaling factor.

In order to make the neuron behave transient chaotic behavior, the parameters are set as follows: $y(1)=0.825, k=0.9, z(1)=0.85, \varepsilon=0.02, I_{0}=0.45, \beta=0.002$,

The state bifurcation figures are respectively shown as Figure 2-Figure 4 when $c=4 . c=5$, $c=7$.
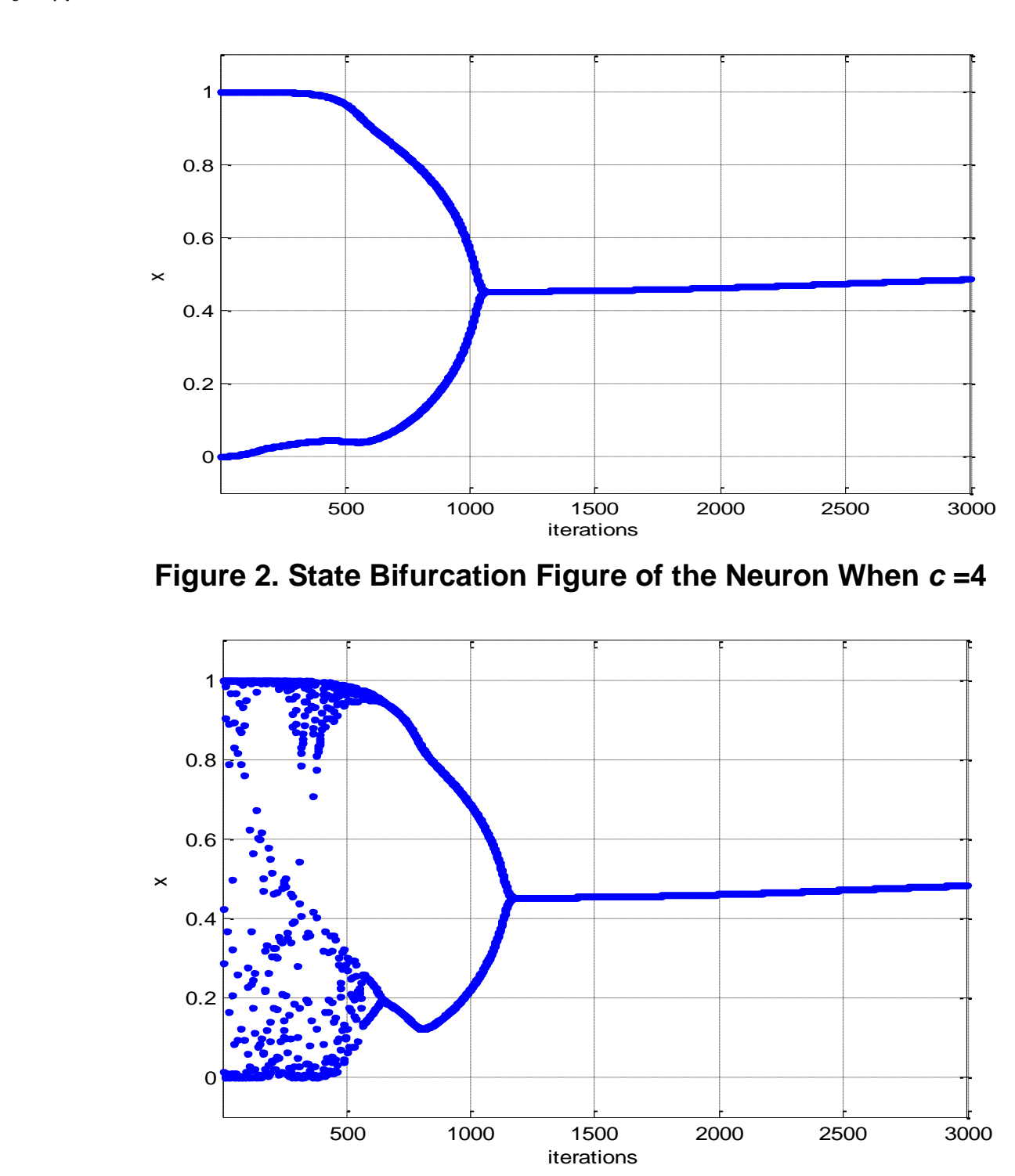

Figure 3. State Bifurcation Figure of the Neuron When $c=5$ 


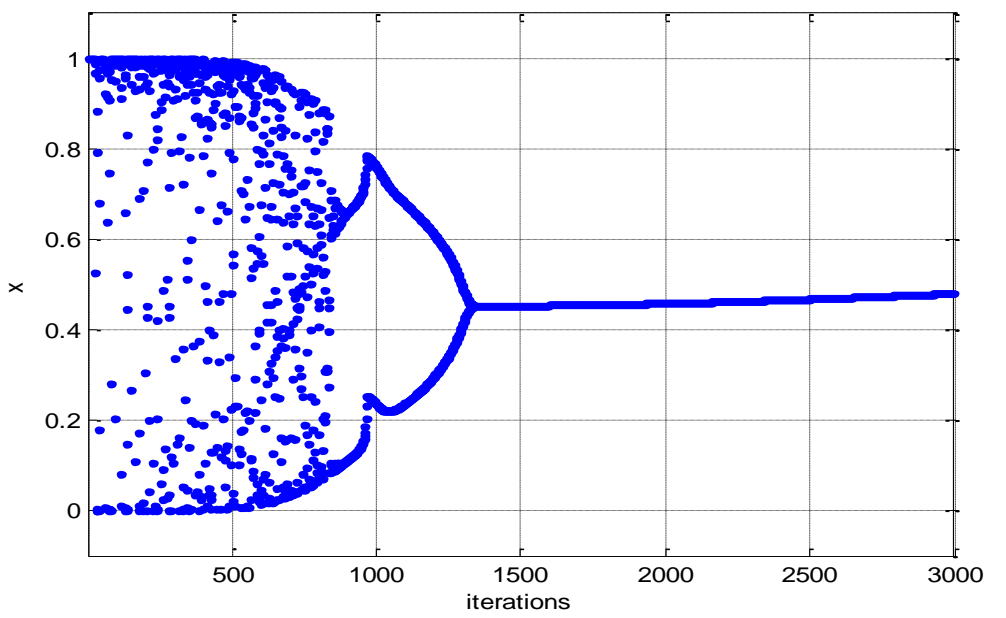

Figure 4. State Bifurcation Figure of the Neuroh When $c=7$

For easy comparison, we take the same parameters. $y(1)=0.825, k=0.9, z(1)=0.85, \varepsilon=0.02, I_{0}=0.45, \beta=0.002$.

The state bifurcation Figures of the Chen-Aihara's network is shown as Figure 5.

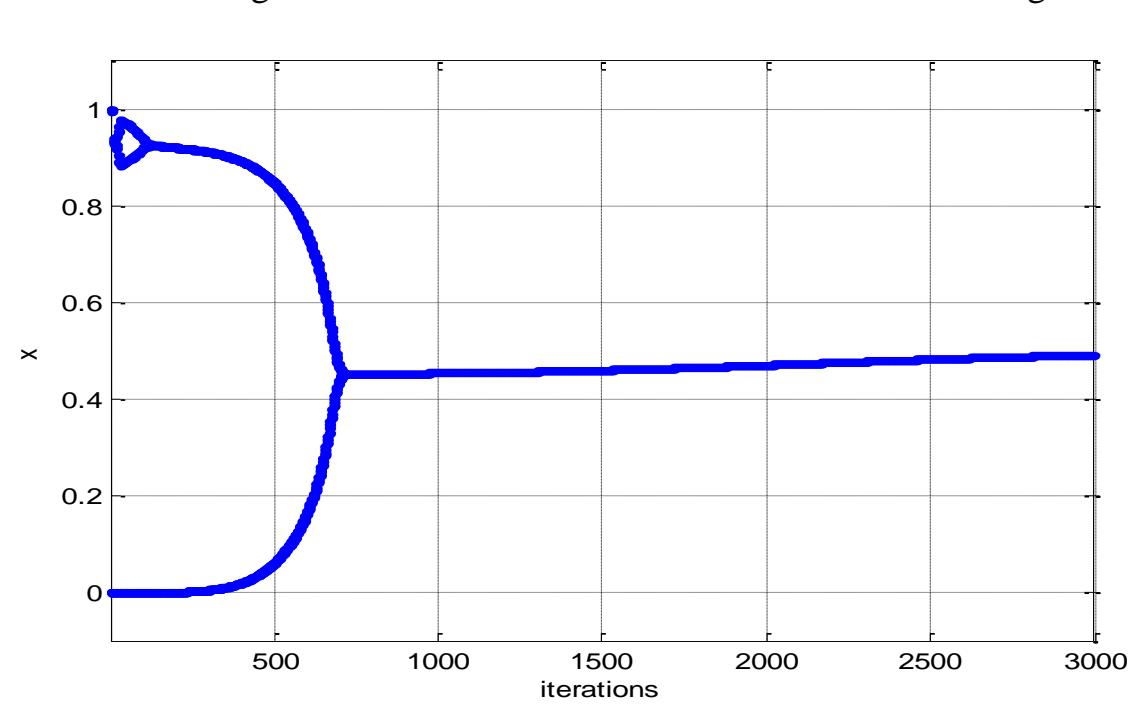

Figure 5. State Bifurcation Figure of the Chen-Aihara's Neuron

We can draw following conclusions by above figures:

(1) Our neural network model has transient behavior of chaotic dynamics. When $z(t)$ decay continuously, the network will gradually become stable equilibrium point through a chaotic bifurcation process, it is easy to know, in the other parameters remain unchanged, when stretching coefficient is about $\mathrm{c}=7$, chaos has the most sophisticated search capabilities.

(2) Comparison with the chaotic neuron model of Chen-Aihara, when the chaotic neuron model to obtain the appropriate scaling factor, Bessel function of the feedback we have created has a more complex chaotic search behavior. 


\subsection{Chaotic Neuron Model of Bessel Function Self-feedback}

Let

$$
\begin{aligned}
& x_{i}(t)=\frac{1}{1+\exp \left(-y_{i}(t) / \varepsilon\right)} \\
& y_{i}(t+1)=k y_{i}(t)+\alpha\left\{\sum_{\substack{j=1 \\
j \neq i}}^{n} w_{i j} x_{j}(t)+I_{i}\right\}^{\prime}-z_{i}(t) g\left(x_{i}(t)-I_{0}\right)(i=1, \cdots, n) \\
& g(x)=J_{1}(c x) \\
& z_{i}(t+1)=(1-\beta) z_{i}(t)
\end{aligned}
$$

Where $x_{i}(\mathrm{t})$ is the output of neuron $i ; y_{i}(\mathrm{t})$ is the internal state for neuron $j_{i}(\mathrm{t})$ selffeedback connection weight; $\beta$ is the simulated annealing parameter; $w_{i j}$ is the connection weight from neuron $j$ to neuron $i, w_{i j}=w_{j i}, w_{i i}=0 ; I_{i}$ is he nimput bias of neuron $i ; \varepsilon$ is the steepness parameters of the activation function; $k$ is the damping factor of the nerve membrane, $0 \leq k \leq 1 ; I_{0}$ is a positive parameter; $g\left(x_{i}(t)-I_{0}\right)$ is feedback term; $\mathrm{c}$ is the scale factor of the Bessel function.

When $g(x)=x$, the feedback term is linear, the model is a classic Chen-Aihara chaotic neural network, this paper presents a continuous Bessel function with $v=1$ as nonlinear selffeedback term. Obviously self-feedback term is non-linear. Since the introduction of nonlinear feedback term Bessel funetion of chaotic neural networks to make new dynamic behavior, compared to linear self-feedback term, the chaotic neural network, Bessel functions chaotic neural networks has more complex dynamics behavior, the internal state of the network with a more complex chaotic search behavior.

\subsection{Network Energy Function and Stability Analysis}

According to the unified theoretical framework, the energy function network model is as follows

$$
\begin{aligned}
& E=E_{H o p}+H \\
& E_{H o p}=-\frac{1}{2} \sum_{i}^{n} w_{i,} x_{i} x_{j}-\sum_{i}^{n} I_{i} x_{i}+\frac{\varepsilon}{\tau} \sum_{i}^{n} \int_{0}^{x_{i}} \ln \frac{x}{1-x} d x \\
& H=\lambda \sum_{0}^{n} g\left(x-I_{0}\right) d x=\lambda \sum_{i}^{n} z_{i} x_{i} g\left(\bar{x}_{i}-I_{0}\right)
\end{aligned}
$$

Where $E_{H o p}$ is the Hopfield network energy function, $H$ is additional energy term, energy function for the network model, $W=W^{T}$ and $w_{i i}=0$

Stability is an important characteristic of nonlinear systems, stability analysis can provide a theoretical basis for the convergence of the network. Analysis energy function is an important method of nonlinear stability analysis of complex systems.

Since $J_{1}^{\prime}(x)=J_{0}(x)-J_{1}(x) / x<1$, then $J_{1}^{\prime}(c x)=c J_{0}(c x)-J_{1}(c x) / x<c$ 
The network with given conditions can be achieved asymptotically stable by the literature [7] derivation process.

Theorem 1 The sufficient condition of Bessel functions asymptotically stable is: there is a minimum eigenvalue of the connection weight matrix (diagonal elements $w_{i i}{ }^{\prime}=-\left(\bar{\theta}_{i} z_{i} c\right) / \alpha$ ) that satisfied:

$$
-\lambda^{\prime}<\frac{2 \varepsilon}{\alpha}\left[4\left(1-\theta_{i}\right)+4 k \theta_{i}\right]=\frac{8 \varepsilon}{\alpha}\left[1-(1-k) \theta_{i}\right]
$$

\section{Bessel Function Self-feedback Chaotic Neural Network Applications}

In order to verify the validity of transiently chaotic neural networks with the Bessel functions feedback term, which are used in the function optimization and combinatorial optimization problems.

\subsection{Application to Function Optimization}

Select the following optimization function [10]

$$
f\left(x_{1}, x_{2}\right)=\left(x_{1}-0.7\right)^{2}\left[\left(x_{2}+0.6\right)^{2}+0.1\right]+\left(x_{2}-0.5\right)^{2}\left[\left(x_{1}+0.4\right)^{2}+0.15\right]
$$

Minimum value of function is 0 , the minimum point of function is $(0.7,0.5)$, local minimum point is $(0.6,0.4)$ and $(0.6,0.5)$.

Let $\varepsilon=0.04, k=1, \alpha=0.05, I_{0}=0.45, \beta=0.002, y(1)=0.825, y(2)=0.825, z_{1}(1)=z_{2}(1)$ $=0.85, c=7$. When using Bessel function self-feedback transiently chaotic neural network model to solving optimization function, the evolution of the energy function shown in Figure 6.

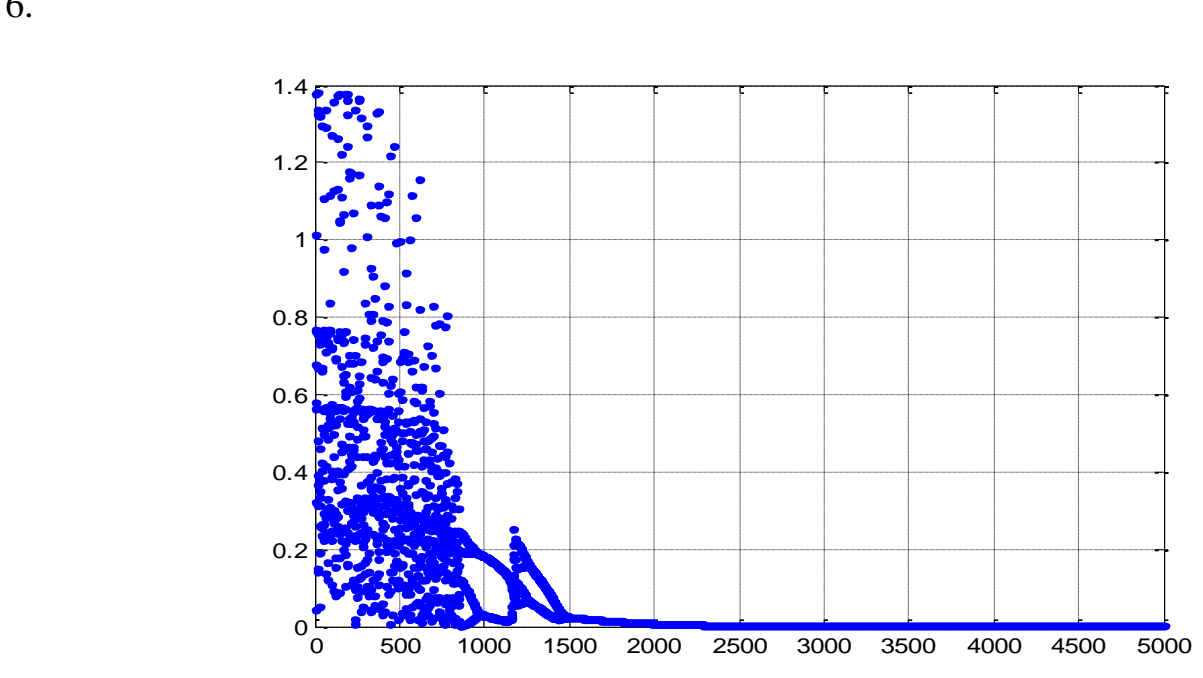

Figure 6. State Bifurcation Figure of our Neuron Network

When our network is running 7000 times, the value of energy function is $1.367386 \times 10^{-11}$ and $x_{1}=0.699997 x_{2}=0.499999$, the time evolution figure of $x_{1}$ and $x_{2}$ is shown by Figure 7 . 

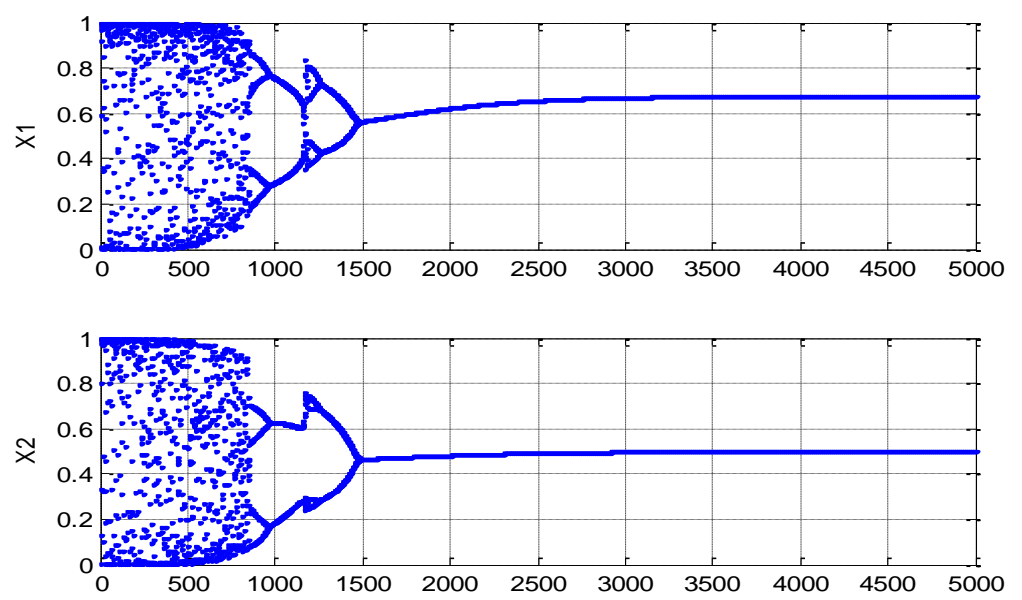

Figure 7. Evolution Figure of $x_{1}$ and $x_{2}$ in our Neural Netvork

In order to compare the Bessel function self-feedback chaotic neural network and ChenAihara chaos neural network model, the parameters are set as follows:

$$
\varepsilon=0.04, k=1, \alpha=0.05, I_{0}=0.45, \beta=0.002, y(1)=0.825, y(2)=0.825, z_{1}(1)=z_{2}(1)=0.85 \text {. }
$$

When Chen-Aihara chaos neural network is running 7000 times, the value of energy function is $2.430467 \times 10^{-12}$ and $x_{1}=0.699998 \alpha_{2}=0.499999$, the time evolution figure of $x_{1}$ and $x_{2}$ is shown by Figure 8 .
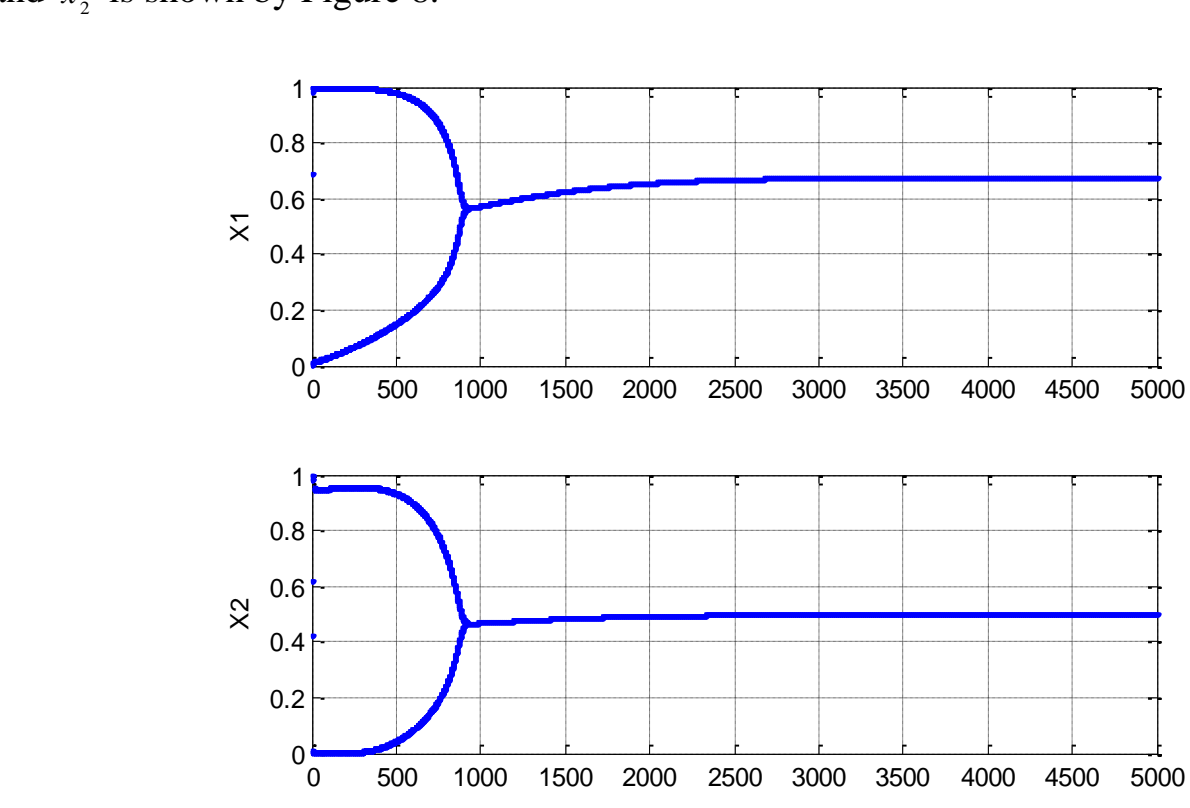

\section{Figure 8. Evolution Figure of $x_{1}$ and $x_{2}$ in Chen-Aihara Chaos Neural Network}

As seen from above, for the continuous functions optimal solution problem, two kinds of neural network model are basically the same solving ability, the chaotic neural network model with Bessel functions the feedback has better search capabilities of chaos, it has a stronger ability to avoid local optima caught. 


\subsection{Application to Combination Optimization (TSP)}

The Traveling Salesman Problem(TSP) is a classically combinational optimization problem and is a NP-hard problem, Symmetric TSP problem with n cities possible have $(n-1) ! / 2$ paths, Find an effective way to solve this problem is the target of many scholars over the years. TSP can be described as follows: To confirm a shortest path and need to visit every city only once when known $\mathrm{N}$ cities and the distance between two cities. This paper will selffeedback Bessel function transiently chaotic neural network model applied to solve the 10 cities TSP problem, simulation results show that this neural network model has a good ability to solve TSP problems.

The energy function takes the follow form [10]:

$$
E=\frac{A}{2} \sum_{i=1}^{n}\left(\sum_{i=1}^{n} x_{i j}-1\right)^{2}+\frac{B}{2} \sum_{j=1}^{n}\left(\sum_{i=1}^{n} x_{i j}-1\right)^{2}+\frac{D}{2} \sum_{i=1}^{n} \sum_{j=1}^{n} \sum_{k=1}^{n} d_{i k} x_{i j}\left(x_{k, j+1}+x_{k, j-1}\right)
$$

Assume $d_{x y}$ to be the distance between city $x$ and $x$ Because the symmetry of the determinant, coefficient $\mathrm{A}=\mathrm{B}$, and a global shortest value of $\mathrm{E}$ expresses a shortest effective path. This paper adopts the following 10-city unitary coordinates: $(0.4,0.4439) ;(0.2439$, $0.1463)$; ( 0.1707, 0.2293); ( 0.2293, 0.716); ( 0.51710.9414); (0.8732, 0.6536); ( 0.6878, $0.5219) ;(0.8488,0.3609) ;(0.6683,0.2536) ;(0.0195,0.2634)$.

The optimal distance from the feedback from the Bessel function transiently chaotic neural network model simulation results is 2.6776 . Figure 9 shows the optimal path.

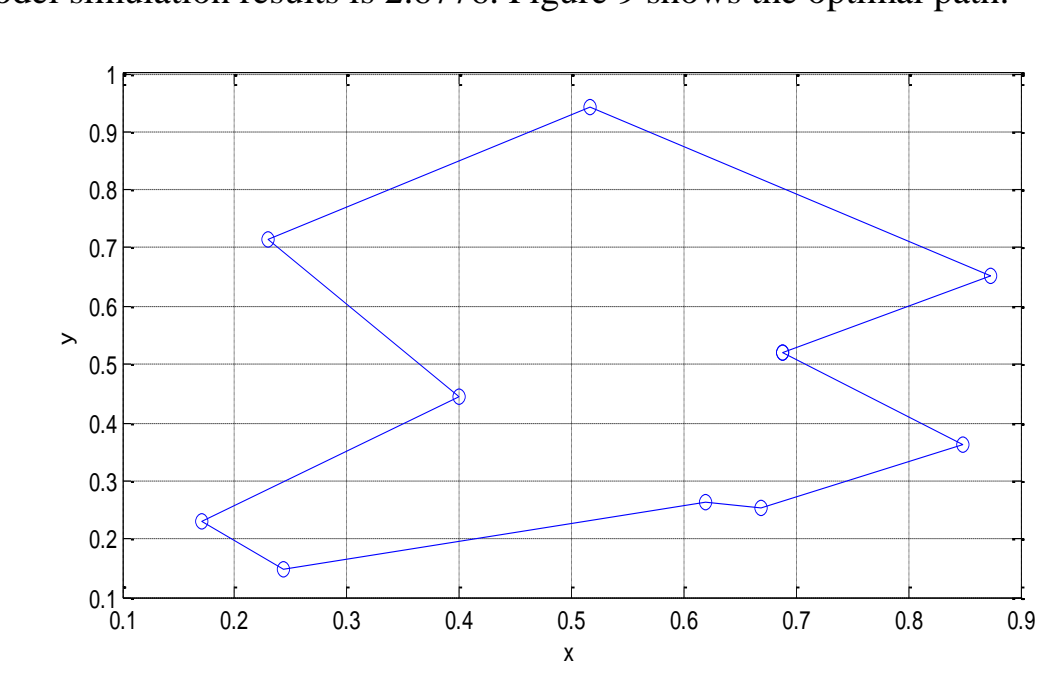

Figure 9. The Optimal Path of 10-city TSP

Select the energy function (15), the parameters of the network are set as follows:

$A=B=1, D=2.5, \alpha=0.08, k=0.9, I_{0}=0.45, \varepsilon=0.04, z(1)=0.8$

The simulation results of 100 different internal conditions with different $\beta$ and $c$ are summarized in Table 1. the column 'NLP', 'NOP', 'RLP', 'RGM' and ANI respectively represents the number of legitimate path, the number of optimal path, the rate of legitimate path, the rate of global minima, the average number of iterations. 
Table 1. Simulation Results with Different $c$ and $\beta$

\begin{tabular}{l|l|l|l|l|l|l}
\hline$c$ & $\beta$ & NLP & NOP & RLP & RGM & ANI \\
\hline \multirow{4}{*}{4} & 0.002 & 88 & 88 & $88 \%$ & $88 \%$ & 512 \\
& 0.003 & 85 & 85 & $85 \%$ & $85 \%$ & 477 \\
& 0.004 & 82 & 81 & $82 \%$ & $81 \%$ & 432 \\
\hline \multirow{5}{*}{5} & 0.002 & 97 & 94 & $97 \%$ & $94 \%$ & 697 \\
& 0.003 & 95 & 93 & $95 \%$ & $93 \%$ & 631 \\
& 0.004 & 94 & 91 & $94 \%$ & $91 \%$ & 546 \\
\hline \multirow{3}{*}{6} & 0.002 & 100 & 100 & $100 \%$ & $100 \%$ & 845 \\
& 0.003 & 99 & 98 & $99 \%$ & $98 \%$ & 763 \\
& 0.004 & 99 & 97 & $99 \%$ & $97 \%$ & 712 \\
\hline \multirow{3}{*}{7} & 0.002 & 100 & 99 & $100 \%$ & $99 \%$ & 1056 \\
& 0.003 & 99 & 96 & $99 \%$ & $96 \%$ & 943 \\
\hline \multirow{2}{*}{8} & 0.004 & 99 & 95 & $99 \%$ & $95 \%$ & 851 \\
\hline & 0.002 & 100 & 55 & $100 \%$ & $55 \%$ & 1385 \\
& 0.003 & 100 & 52 & $100 \%$ & $52 \%$ & 1342 \\
\hline
\end{tabular}

As seen from Table 1:

(1) When stretching factor is 6 or 7 and simulated annealing parameters is $\beta=0.002$, Bessel function self-feedback transiently chaotic neural network model to solve the 10 cities in the traveling salesman problem with the best effect, almos 100 percent to get the optimal path.

(2) The value of the scale factor andsimulated anneallng parameters have a significant impact on the efficiency of the network model to solve: the smaller the coefficient of expansion and contraction, the moechaotic search process is relatively simple algorithms easier to get the minimum value; Conversely, the greater the stretching coefficient, chaos the more complicated the search process, the atgorithm has stronger to get rid of local optimum value reaches the global optinum.

(3) Simulated annealing parameters in the vicinity of 0.003 for optimizing network capacity is not affected, but have a more significant impact on the convergence speed of the network, when the annealing parameters away from 0.003 , the network optimization capability significantly decreased.

\section{Conclusion}

This paper presents a new self-feedback neural network, the feedback chaotic neural network with Bessel functions. Lyapunov stability analysis is performed by using a unified theoretical framework, the results show that the neural network model under the given condition can reached asymptotically stable, when you select the proper stretching Department, the solving process of optimization problems and the traveling salesman problem show that this neural network model has more to avoid falling into local minima network capacity, which can more effectively improve optimization capabilities of the network. 


\section{References}

[1] L.-N. Chen and K. Aihara, "Chaotic Simulated Annealing by a Neural Network Model with Transient Chaos", Neural Networks, vol. 8, no. 6, (1995), pp. 915-930.

[2] Y. Q. Xu and M. Sun, "Wavelet Chaotic Neural Network and its Application in Optimization Calculation, The 6th World Congress on Intelligent Control and Automation (WCICA2006), Dalian, (2006), pp. 30043009.

[3] L. Zhao, M. Sun, J. H. Cheng and Y. Q. Xu, "A Novel Chaotic Nrural Network With the Ability to Characterize Local Features ang Its Application”, IEEE Trans. on Neural Networks, vol. 20, no. 4, (2009), pp. 737-738.

[4] Y. Q. Xu and M. Sun, "Shannon Wavelet Chaotic Neural Network to Solve TSP Problems", Control Theory and Applications, vol. 25, no.3, (2008), pp. 574-577.

[5] X. Chunbo and L. Xiangdong, "A New Chaotic Neural Network and its Application", Acta Electronica Sinica, vol. 33, (2005), pp. 868-870.

[6] H. Yuyao and W. Libo, "Chaotic Neural Network and its Application in Optimization Problems Control Theory \& Applications, vol. 17, no. 6, (2000), pp. 847-852.

[7] Y.Q. Xu and X. Yang, "Chaotic Neural Network with Inverse Trigonometric Functions Feed ack and its Application”, Journal of Harbin University of Commerce, vol. 26, no. 3, (2010), pp. 324-328.

[8] Research of Nonlinear Self-feedback Chaotic Neural Network, Harbin Engineering University, (2011), pp. 25-36.

[9] M. Sun, "Chaotic Neural Network and its Application Based on Wavelet and Hysteresis-Harbin Engineering University, (2010), pp. 39-45.

[10] Y.-Q. Xu, M. Sun, "Chaotic Neural Network and Its Application", Heilongiiang University.

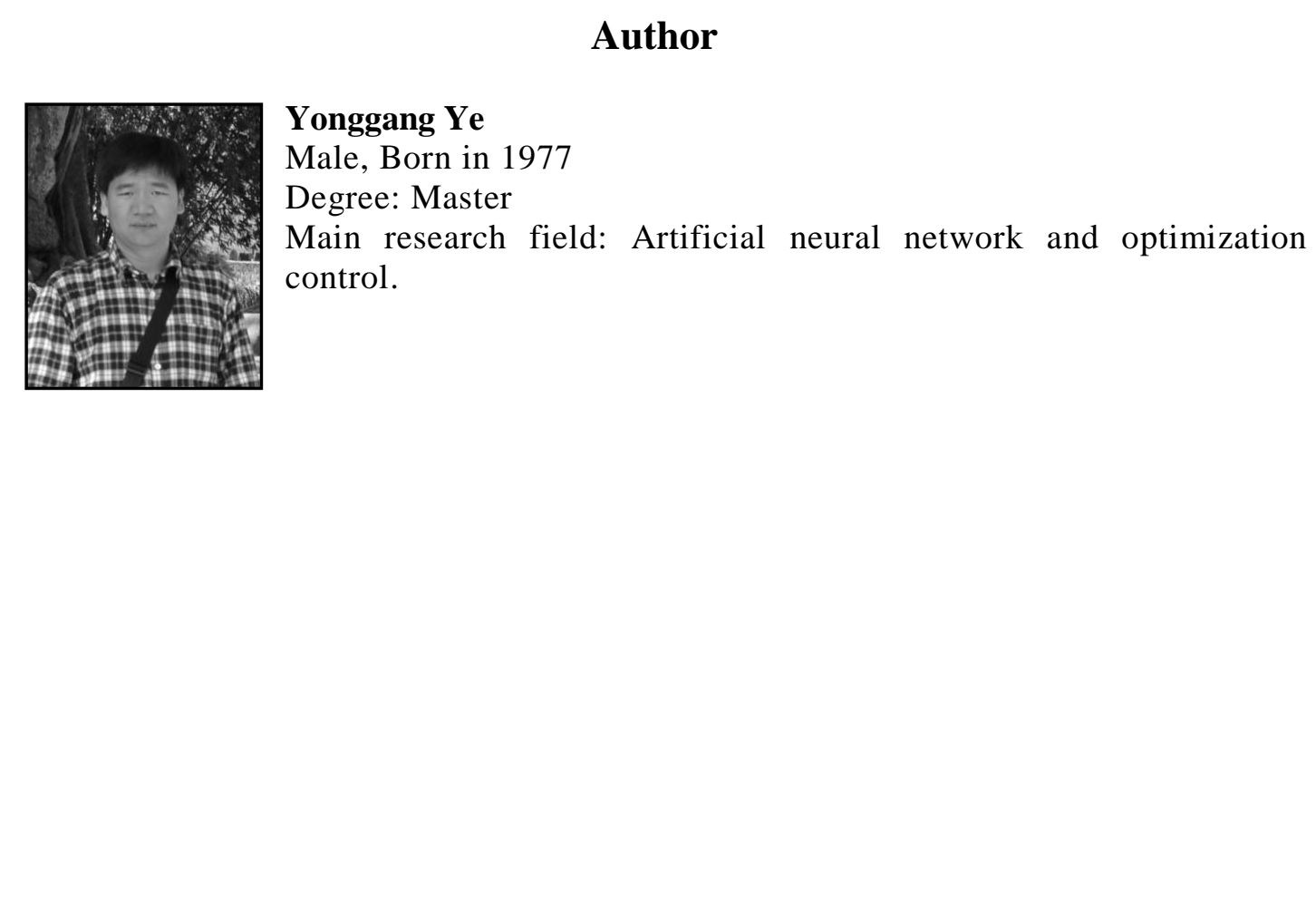

